\title{
What makes time different from space?*
}

\author{
Bradford Skow
}

\section{Introduction}

No one denies that time and space are different; and it is easy to catalog differences between them. I can point my finger toward the west, but I can't point my finger toward the future. If I choose, I can now move to the left, but I cannot now choose to move toward the past. And (as D. C. Williams points out) for many of us, our attitudes toward time differ from our attitudes toward space. We want to maximize our temporal extent and minimize our spatial extent: we want to live as long as possible but we want to be thin. ${ }^{1}$ But these differences are not very deep, and don't get at the essence of the difference between time and space. That's what I want to understand: I want to know what makes time different from space. I want to know which difference is the fundamental difference between them.

I will argue for the claim that (roughly) time is that dimension that plays a certain role in the geometry of spacetime and the laws of nature. (In this paper, then, I focus on what is distinctive about time, and say little about what is distinctive about space.) But before giving the argument I want to put my question in slightly different terms. Instead of asking, 'what makes time different from space?, I want to ask, 'what makes temporal directions in spacetime temporal, rather than spatial?'2 After rejecting some bad answers to this question I'll present my view.

*Published in Nô̂s 41 (2007): 227-252. Caution: the numbering of the figures in this version is different from the numbering in the published version.

${ }^{1}$ [Williams 1951: page 468]. Williams actually says that we care how long we live but do not care how fat we are.

${ }^{2}$ It is important to distinguish this question from another commonly discussed 


\section{Spacetime Diagrams and Directions in Spacetime}

It is often helpful, when approaching problems in physics and in metaphysics, to draw a spacetime diagram. Spacetime diagrams represent the careers in space and time of some material objects. Traditionally in a two-dimensional spacetime diagram (the easiest kind to draw on paper) the horizontal axis represents space and the vertical axis represents time. So suppose I'm confined to one dimension in space: I can only move to the left or to the right. Then the diagram on the left of figure 1 might represent part of my career. The zig-zag line represents me; it's my worldline. (I'm incorrectly represented as point-sized, but that's not important.) According to the diagram I stand still for a while; then I walk to the left, stop, stand still for a little while longer, and then walk back to the right.

I said I wanted to ask what makes temporal directions in spacetime temporal, rather than spatial. So what is a direction in spacetime? We can use spacetime diagrams to get a sense for what directions in spacetime are. To represent a direction in spacetime (at some spacetime point) on the diagram we can draw an arrow, or vector, on the diagram at the point that represents that point of spacetime. So in the diagram on the right of figure 1 the arrow labeled ' $A$ ' points in the leftward direction in space and the arrow labeled 'B' points in the future direction in time. There are in this diagram, then, at least two temporal directions: toward the future and toward the past; and two spatial directions: toward the left and toward the right.

Two arrows may point in the same direction while being of different lengths. A direction then is an equivalence class of vectors-the set of all vectors that point in the same direction and differ only in their length. Following standard usage, I will sometimes call a vector that points in a temporal direction a 'timelike vector,' and a vector that points in a spatial direction a 'spacelike vector.'

What about the arrows labeled ' $C$ ' and ' $D$ '? They don't seem to point in either question. Many philosophers want to know what makes the future different from the past. But that is not what I am asking. Toward the future and toward the past are both temporal directions, and I am not asking what makes one temporal direction the direction toward the future and the other, the direction toward the past. Instead I'm asking, what makes either of them a temporal, rather than spatial, direction in the first place? 
Figure 1: Two Spacetime Diagrams
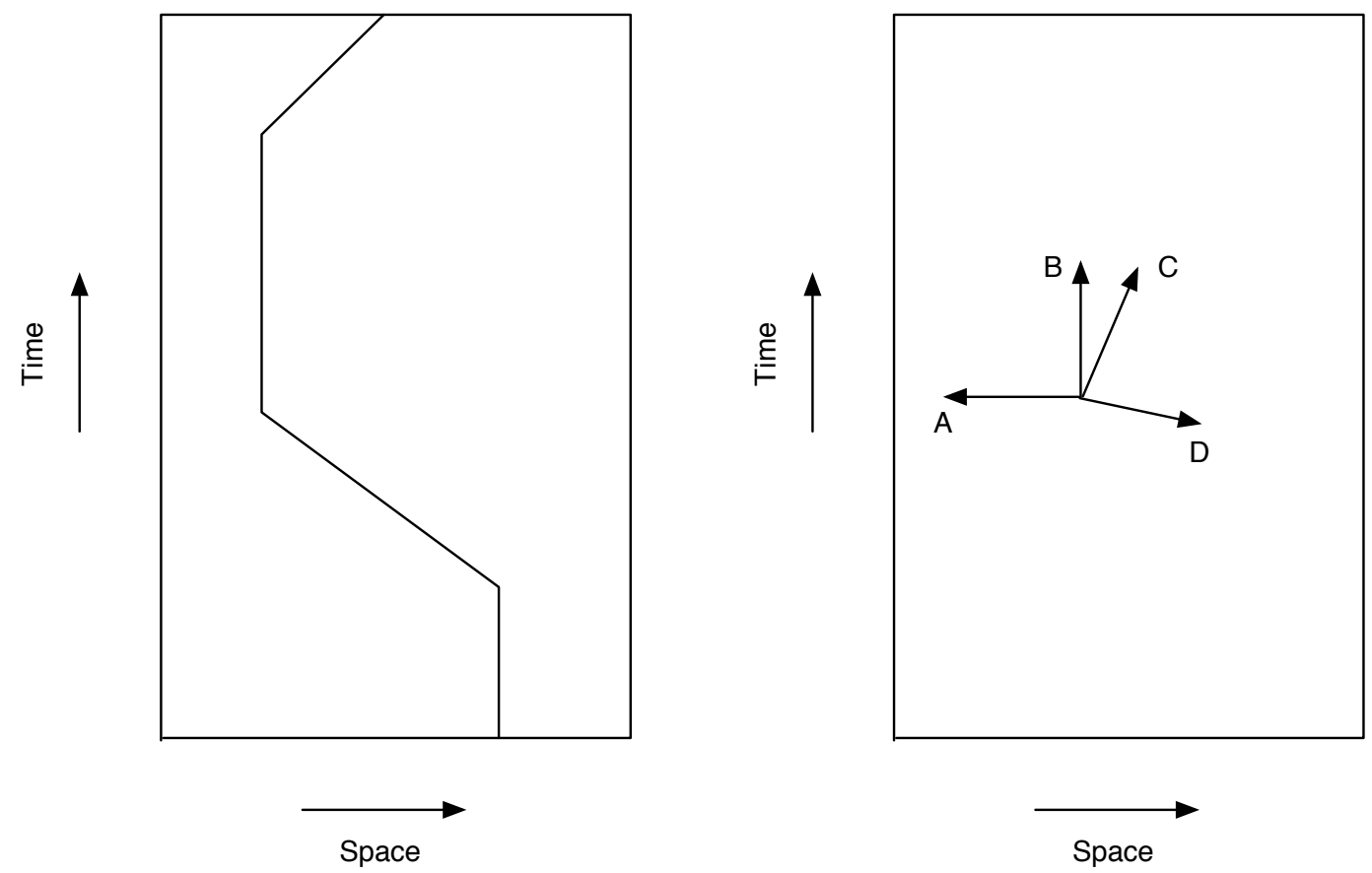

a temporal or a spatial direction. What to say about arrows like C and D really depends on what geometrical structure the spacetime represented by the diagram has. In (two-dimensional) neo-Newtonian spacetime every arrow that does not point either to the left or the right points in a temporal direction, while in Minkowski spacetime (the spacetime of the special theory of relativity) arrows that are less than 45 degrees from the vertical (like $\mathrm{C}$ ) point in a temporal direction, while arrows that are more than 45 degrees (like D) from the vertical point in a spacelike direction.

Why frame the discussion in terms of spatial and temporal directions, rather than space and time? Modern physical theories are formulated in terms of a fourdimensional spacetime, instead of in terms of three-dimensional space and onedimensional time separately. In some older theories (Newtonian mechanics, in particular) there is a way to identify certain regions of spacetime as points of space and other regions as instants of time. But in more recently theories, especially the 
general theory of relativity, this cannot always be done.

We can identify points of space and instants of time with certain regions of Newtonian spacetime because Newtonian spacetime has certain special geometrical features (see figure 2).

Figure 2: Newtonian Spacetime

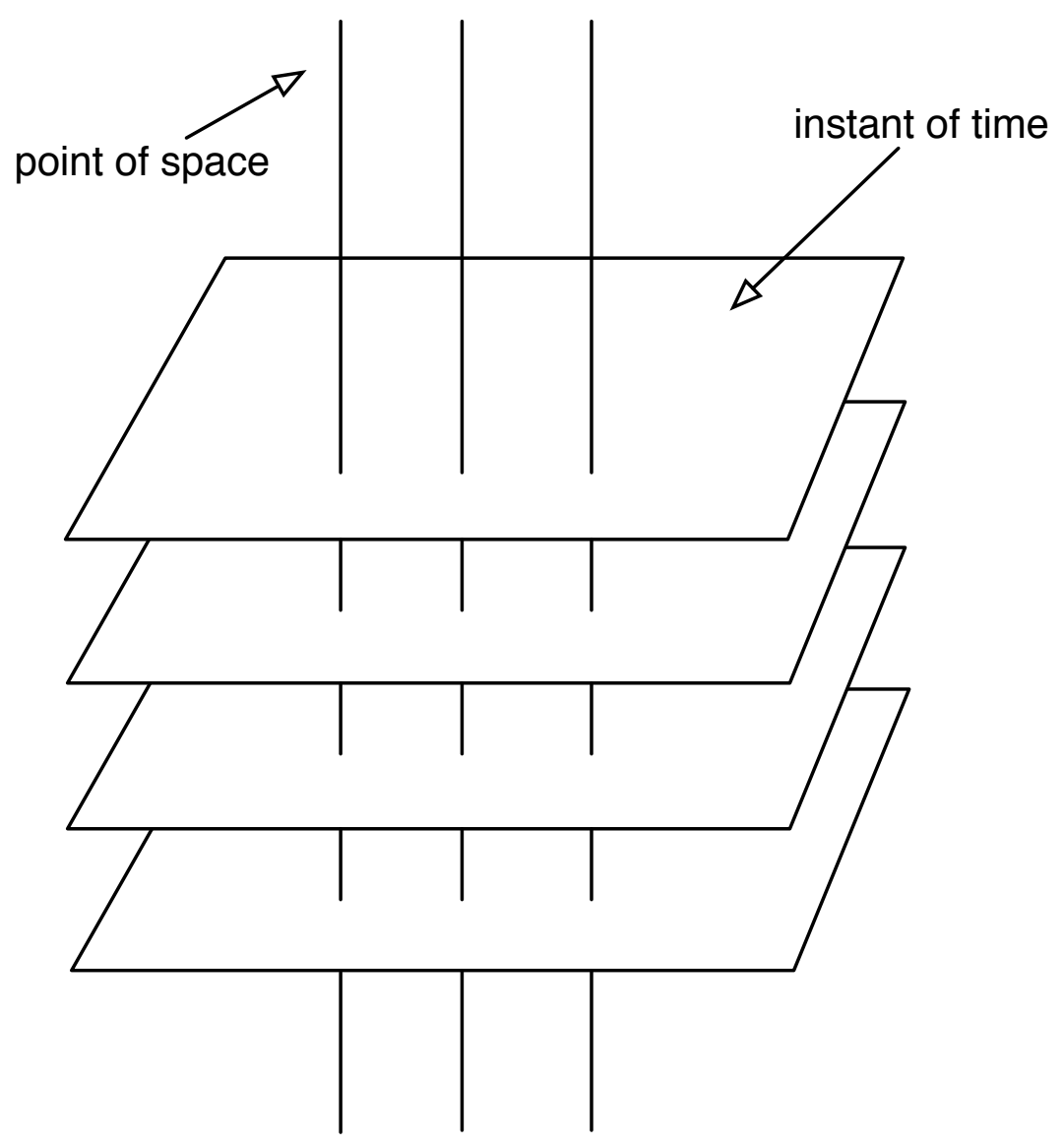

There is a unique and geometrically preferred way to divide up this four-dimensional spacetime manifold into a sequence of three-dimensional Euclidean submanifolds. Each of these Euclidean submanifolds is well-suited to play the role we want instants of time to play: events that occur on the same submanifold occur simultaneously. So these submanifolds are instants of time. A point of space, then, is a line 
in spacetime perpendicular to each time. (Events located on the same line, whether simultaneous or not, occur in the same place.) Facts about which regions are points of space, and which are instants of time, are absolute: not relative to any observer or frame of reference.

In Newtonian spacetime the distinction between time and space and between temporal and spatial directions coincide. A vector that points in a spatial direction is one that points along (is tangent to) a time, and so points toward other points of space. A vector that points in a temporal direction is one that points at an angle to a time, and so points in the direction of future or past times.

But the special geometrical features that allow us to identify points of space and instants of time with certain regions of Newtonian spacetime are missing in other spacetimes. In neo-Newtonian spacetime, though there is a non-relative way to identify regions of spacetime with times, there is not a non-relative way to identify regions of spacetime with points of space. Different inertial observers will regard different events as occurring in the same place, and so will regard different lines in spacetime as points of space, without the geometry of spacetime privileging one of them over the others. ${ }^{3}$ This happens to time as well in Minkowski spacetime, the spacetime of special relativity.

In general relativity it gets worse. According to this theory the geometry of spacetime varies from world to world, depending on the distribution of matter in each world. In some of those worlds spacetime can be divided up into instants of time and points of space. (As before the times are three-dimensional submanifolds having certain geometrical properties, but in general relativity they need not have a Euclidean geometry. ${ }^{4}$ Points of spaces are curves in spacetime (they need not be straight lines) that run oblique to each time and meet some further geometrical conditions. Typically there are many ways to divide spacetime up, just as in Minkowski spacetime, none more preferred by the geometry than the others.) But

\footnotetext{
${ }^{3}$ I said above that in Newtonian spacetime a point of space is a line in spacetime that intersects each time at a right angle. In neo-Newtonian spacetime we can no longer say which lines intersect each time at a right angle and which do not.

${ }^{4}$ Technically, the relevant geometrical property is that these submanifolds are spacelike.
} 
in other worlds spacetime cannot be divided in this way at all. (Gödel's solution is an example.) In these worlds no regions of spacetime count as points of space or instants of time, not even relative to some inertial observer. But even in these worlds there is a distinction between the temporal and the spatial aspects of spacetime, because we can still distinguish spacelike from timelike directions. (Given a point on the worldline of a conscious observer in one of these spacetimes, for example, we can still tell in which directions from that point his future mental episodes lie.) Since the distinction between directions is more general than the distinction between space and time, it is the distinction on which I want to focus.

\section{Does Geometry Distinguish Temporal from Spatial Directions?}

The view that the distinction between temporal and spatial directions is a geometrical one is a natural one to take when one studies spacetime theories. Those theories attribute one or another geometrical structure to spacetime, and when a given theory is explained, the distinction between temporal and spatial directions is usually explained in geometrical terms.

For example, according to the special theory of relativity, the geometry of spacetime satisfies the axioms of Minkowski geometry. That geometry allows us to assign lengths to vectors in spacetime. And the lengths of vectors that point in temporal directions have a different sign than the lengths of vectors that point in spatial directions. One kind has negative lengths; the other, positive.

We might hope to explain what makes temporal directions temporal by appealing to the signs of the lengths of vectors that point in those directions; but I don't see how this approach could work, for three reasons.

First, while looking at the signs of the lengths of the vectors may allow us to pick out two disjoint classes of vectors (namely, the class of vectors with negative lengths, and the class of vectors with positive lengths), the sign of the length of a vector does not fix whether it is a timelike vector. Consider a world $w$ that is just like ours except that the sign of the length of every vector has been switched. If timelike vectors are (say) vectors with negative lengths, then $w$ is very different from the actual world: vectors that are actually timelike are spacelike in $w$. But 
this is not what textbooks on relativity say about $w$. They say that $w$ is qualitatively indiscernible from our world: the actual timelike vectors are still timelike in $w$, even though their lengths are negative in the actual world and positive in $w .{ }^{5}$

Second, a difference in the signs of their lengths is too formal and abstract to be the fundamental difference between vectors that point in temporal and those that point in spatial directions, and so between time and space.

And third, distinguishing between the two kinds of directions in terms of the signs of vectors' lengths only works in spacetimes with Minkowski geometry. No vectors have negative lengths in neo-Newtonian spacetime, but we do not want to say that no directions in that spacetime are temporal. Of course in that spacetime the geometrical distinction between spatial and temporal directions is explained differently. Roughly speaking, in neo-Newtonian spacetime we have two ways to

\footnotetext{
${ }^{5}$ What the textbooks say is actually more complicated. They say that whether timelike vectors have negative length or positive length is a matter of convention. I understand this in the following way. The textbooks use mathematical objects to represent spacetime: a differentiable manifold with a flat Lorentz metric. But different books use different metrics. Like spacetime itself, a differentiable manifold has vectors at each of its points, and the metric assigns lengths to these vectors. The textbooks that say that timelike vectors have negative lengths use a different metric from the textbooks that say that timelike vectors have positive lengths. The metrics disagree only over the signs of lengths of vectors. But this difference makes no difference to the geometrical structure of the manifolds: the two manifolds have the same geometrical structure. So when textbooks say that whether timelike vectors have negative length or positive length is a matter of convention, they mean that these two manifolds are equally good representations of the geometrical structure of spacetime itself.

This entails the claim I attribute to the textbooks in the text: that $w$ is qualitatively indiscernible from the actual world. The argument goes like this. If two abstract differentiable manifolds that differ only over the signs of the lengths of their vectors have the same geometrical structure, then two concrete spacetimes (like the actual spacetime and the spacetime in $w$ ) that differ only over the signs of the lengths of their vectors also have the same geometrical structure. So any vector that actually has negative length has all the same geometrical features in the actual world as it does in $w$, where it has positive length. Assuming (as I did in the text) that $w$ also agrees with the actual world on all of its non-geometrical features, it follows that the two worlds are qualitatively indiscernible.
} 
assign (positive) lengths to vectors. We are told that vectors that point in timelike directions are those that have non-zero length according to a particular one of those ways. (The same is true in Newtonian spacetime.) But it won't do to say that what makes a timelike vector timelike is that is satisfies the following condition: either it is a vector in Minkowski spacetime and it has (say) negative length, or it is a vector in neo-Newtonian spacetime and it has positive length according to one particular metric (and so on with clauses for each different spacetime). For temporal directions in a world with one spacetime geometry have something in common with temporal directions in a world with some other spacetime geometry. And an answer to the question, 'in virtue of what are temporal directions temporal?', must tell us something about what they have in common. Even if the current proposal correctly distinguishes temporal from spatial directions, it doesn't say what temporal directions have in common. So it is not the answer we're looking for.

While we have not yet found a geometrical way to distinguish timelike from spacelike directions, we do have a geometrical way to distinguish directions that are either spacelike or timelike from those that are neither. In all spacetimes vectors that are either spacelike or timelike have non-zero length. And we can establish that vectors with zero length are neither spacelike or timelike on geometrical grounds alone. ${ }^{6}$ Whichever spacetime geometry we look at, that geometry assigns lengths to vectors as a way to assign distances, either spatial or temporal, between spacetime points. (This length is determined by adding up (really, integrating) the lengths of vectors tangent to a certain path between the two points.) But adding up a bunch of zeros just gives zero, so adding up the lengths of vectors with zero length couldn't be a way to determine the temporal or spatial distance between two points. So vectors with zero length are neither spatial nor temporal.

We have, then, a geometrical way to divide the vectors in any spacetime into the class of vectors that are either spacelike or timelike, and the class of vectors that are neither. We also have a geometrical way to divide the class of vectors that

\footnotetext{
${ }^{6}$ The zero vector, which points in no direction at all, has zero length, but in some spacetimes other vectors do as well. In particular, 'lightlike' vectors in Minkowski spacetime have zero length. (These vectors point along possible paths of light rays.)
} 
are either spacelike or timelike into two subclasses. In Minkowski spacetime (and in the spacetimes of general relativity as well) we divide them into the subclass with negative lengths and the subclass with positive lengths. In Newtonian and neo-Newtonian spacetime we divide them into the subclass with positive length according to one way of assigning lengths, and the subclass with positive lengths according to the other way of assigning lengths. (Let's say that two vectors that belong to the same subclass are 'of the same kind.' Talk of vectors that are of the same kind, then, is reserved for vectors that are either spatial or temporal.) But we don't yet have a way to designate one of those subclasses as the class of vectors that point in temporal directions.

\section{Dimensionality}

If we're looking for a geometrical way to distinguish temporal from spatial directions, dimensionality considerations are probably our best bet. In four-dimensional Minkowski geometry, whether timelike vectors have positive or negative lengths, time is one-dimensional and space is three-dimensional. Perhaps it is because it is one-dimensional that time is time.

Before examining this thesis I'll say something about what it means to say that time is one-dimensional.

Intuitively speaking, to say that time is one-dimensional is to say that we can represent time as a line, and that all events that occur in time can be assigned a position on that line. ${ }^{7}$

As I said above in section 2, in Newtonian and neo-Newtonian spacetime there is a unique geometrically preferred way to slice up the four-dimensional spacetime into a one-dimensional sequence of three-dimensional Euclidean submanifolds. Each three-dimensional submanifold is a time, and the sequence gives their temporal ordering. Since every event occurs somewhere in spacetime, every event occurs somewhere in this one-dimensional sequence. So time is onedimensional in the intuitive sense in these spacetimes because it divides up in this

\footnotetext{
${ }^{7}$ Circles are one-dimensional too, so strictly speaking time could be one-dimensional even if we had to represent time as a circle.
} 
way.

In Minkowski spacetime there are many geometrically preferred ways to slice up the four-dimensional spacetime into a sequence of three-dimensional Euclidean submanifolds. We can still, then, temporally order all events on a line, even though there is no unique way to do so. (There are events $x$ and $y$ such that $x$ occurs before $y$ according to one slicing but $y$ occurs before $x$ according to another slicing.)

But we do not want to say in general that time is one-dimensional just in case the geometry of spacetime gives us at least one special way to divide it into a one-dimensional sequence of three-dimensional submanifolds. For, as I mentioned, some general relativistic spacetimes cannot be divided up naturally into a sequence of three-dimensional submanifolds. And there is still a (somewhat technical) sense in which time is one-dimensional in worlds containing those spacetimes.

The vectors in spacetime at any given spacetime point form a four-dimensional vector space; the maximum dimension of a subspace containing only timelike vectors (and the zero vector) is one, while the maximum dimension of a subspace containing only spacelike vectors (and the zero vector-I'll leave this implicit from now on) is three. ${ }^{8}$ Time is one-dimensional in this more technical sense not just in general relativistic spacetimes, but also in the other spacetimes I've mentioned; so this more technical sense is a generalization of the one I gave earlier.

To see why this is so in Minkowski spacetime, consider (for ease of visualization) three-dimensional Minkowski spacetime, depicted in figure 3. From a given point in that spacetime the set of points that can be reached by light rays forms a double cone: the future and past light cones at that point. The set of vectors that lie inside either light cone are the timelike vectors. If you pick any line through that cone and consider the set of vectors that point along that line, then (as I explained in note 8 ) those timelike vectors form a one-dimensional subspace of the space of all vectors at that point. But there couldn't be a two-dimensional subspace of timelike

\footnotetext{
${ }^{8}$ Here's a brief, intuitive explanation of vector spaces. Think of a (three-dimensional) vector space as the set of all arrows that can be drawn (in ordinary space) from a point. The arrow of zero length counts: it's the zero vector. A subspace of that vector space is a subset of the arrows such that either all of the arrows lie in the same plane (that's a two-dimensional subspace) or all of the arrows lie on the same line (that's a one-dimensional subspace).
} 
Figure 3: Minkowski Spacetime

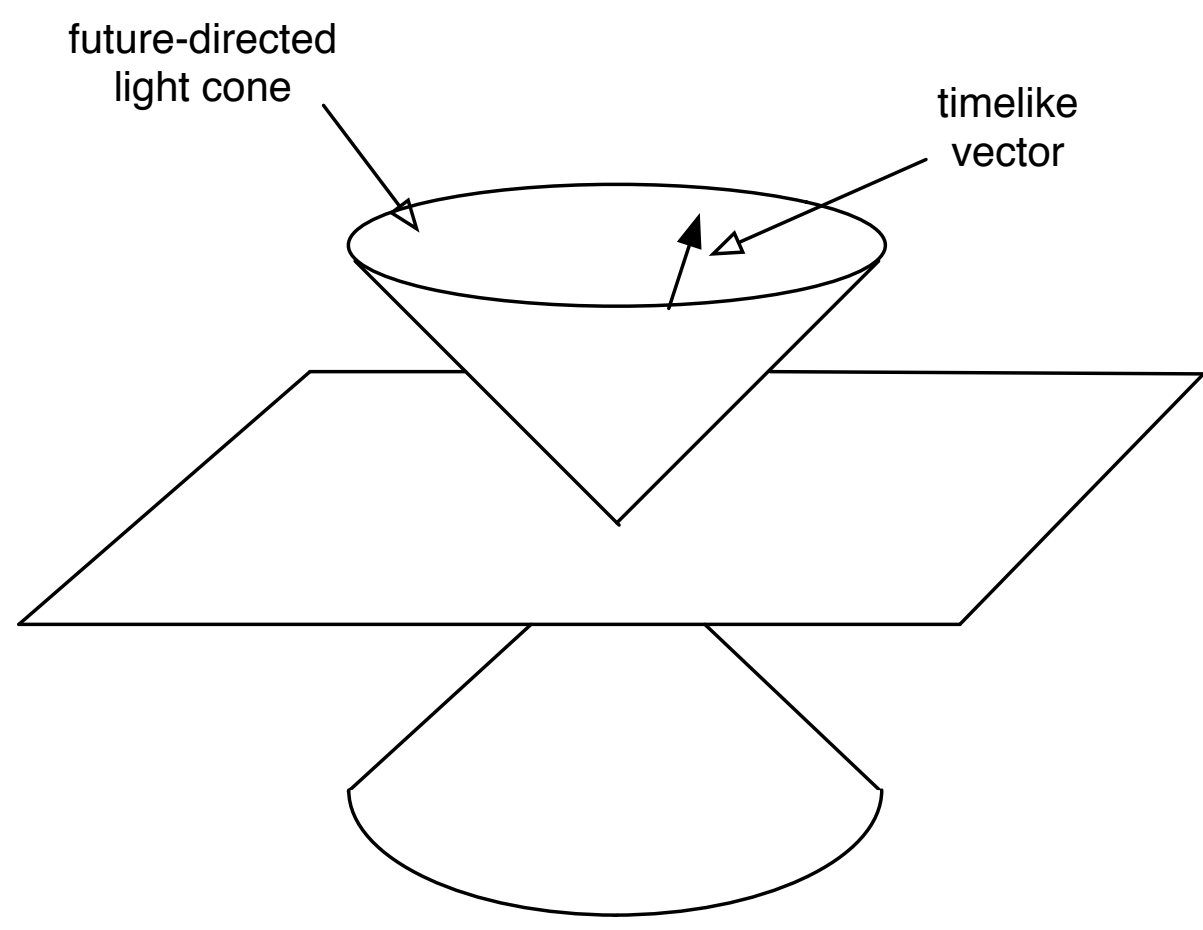

vectors: if there were, then there would have to be some plane through that point in spacetime such that all the vectors at that point that lie in that plane also fall inside either the past or future light cone. But no plane lies entirely inside the two light cones.

It's not controversial that time is one-dimensional in the familiar spacetime theories. The controversial claim is that it is dimensionality that makes timelike vectors timelike. To be explicit, the controversial claim is:

Dimension: A vector is timelike iff the maximum dimension of a subspace containing only vectors of the same kind as it is one. ${ }^{9}$

\footnotetext{
${ }^{9}$ In Minkowski spacetime the maximum dimension of a subspace containing only null vectors (lightlike vectors and the zero vector) is one. But DimENsion does
} 
I objected above to the first way of using geometry to distinguish spatial from temporal directions that it didn't tell us what temporal directions in different spacetimes had in common. Dimension does better, because it does say something about what they have in common.

There are, however, problems for Dimension. First, the dimensionality difference between time and space doesn't seem deep enough to be the fundamental difference between the two. I take it that the fundamental difference between space and time will illuminate the other, less fundamental, differences between them, and help us explain those other differences. But the difference in dimensionality doesn't do this.

Second, and more important, it seems possible that both time and space be one-dimensional. But in a possible world with two-dimensional Minkowski spacetime, all the (non-null) vectors meet the condition in Dimension. So Dimension entails that all (non-null) directions in that world are temporal, and so (since no direction is both spatial and temporal) that that world contains no spatial directions at all. But that can't be right: surely it's possible that special relativity be true and that time and space both be one-dimensional.

The problem is that while vectors of one kind satisfy the condition in DimensIon, vectors of the other kind do as well; while we have already established that all timelike vectors are of the same kind. Someone who maintains that dimensionality is the only factor that does any work to distinguish timelike from spacelike directions, then, must admit that the condition in Dimension is necessary but not sufficient for a direction to be timelike; and that no condition is sufficient in every world. He might then revise his view as follows:

DiMENSION*: If a vector is timelike then the maximum dimension of a subspace containing only vectors of the same kind as it is one; all timelike vectors are of the same kind; and to the extent that these conditions

not entail that these vectors are timelike. In section 3 I argued that null vectors are not timelike on geometrical grounds alone; Dimension, like the other principles I will later advance, is only meant to determine which of the remaining vectors are timelike. (Recall I said in section 3 that talk of two vectors being of the same kind is reserved for vectors that (unlike null vectors) are either spatial or temporal.) 
fail to determine which vectors are timelike, it is to that extent indeterminate which vectors are timelike.

In worlds where just one kind of vector satisfies the condition in DimENsion, then, Dimension and Dimension* agree that vectors of that kind are timelike. But in worlds with two-dimensional spacetimes Dimension* entails that it is indeterminate which kind of vector is timelike.

In the end, though, this move to indeterminacy fails. It fails not because I insist that it must be perfectly determinate in every world which directions are timelike. But surely in some two-dimensional worlds, complicated worlds in which plenty is happening, there is a fact of the matter about which directions are temporal. So we should reject Dimension* as well as DimEnsion.

I have looked at two ways to distinguish temporal from spatial directions in geometrical terms, and found reasons for rejecting both. Might some other attempt to distinguish them in geometrical terms succeed where these have failed? The answer is 'no.' The two-dimensional spacetimes that make trouble for DimENsion also make trouble for any attempt to use geometry alone to distinguish temporal from spatial directions. For the roles that timelike and spacelike directions play in the geometry of two-dimensional Minkowski spacetime (and two-dimensional Newtonian spacetime) are symmetric. Since timelike and spacelike directions play symmetric roles in the two-dimensional spacetime geometries, any attempt to distinguish temporal from spatial directions by isolating a geometrical role that one but not the other plays is bound to fail.

So what else other than or in addition to the geometry of spacetime makes the difference between spacelike and timelike directions?

\section{Laws of Nature}

Timelike and spacelike directions play different roles in the laws of physics that we have taken seriously as the fundamental laws governing our world. Those laws govern the evolution of the world in timelike directions, but not in spacelike directions.

This claim might look analytic ('of course evolution happens in time'), but 
I'm using 'govern the evolution of the world' in a stipulated sense that does not build time into its meaning. Roughly speaking, by 'the laws govern the evolution of the world' in some direction I mean that the laws, together with complete information about what is going on in some region of spacetime, yield complete information about (or assign probabilities to complete descriptions of) what is going on in regions of spacetime that lie in that direction from the initial region.

My meaning can be made more precise using an example. Earlier I said that the spacetimes of Newtonian mechanics and special relativity, as well as some of the spacetimes of general relativity, can be partitioned into a sequence of times-a sequence of (geometrically special) three-dimensional submanifolds. Now in Newtonian gravitational theory, given complete information about what is going on on one time, the laws determine what is going on on the rest of the times. ${ }^{10}$ These laws govern the evolution of the world from one time to the others. And a similar claim is true for other laws we've considered fundamental.

Now, timelike vectors are not tangent to any time, on any way of partitioning any given spacetime into times. Rather, no matter which partitioning of spacetime into times you use, timelike vectors point from one time toward others. So timelike vectors point in the directions in which the laws govern the evolution of the world. ${ }^{11}$

The same is not true of points of space. If I know what is going on right here (at this location in space) for all time, the laws do not give me complete information about (or assign probabilities to complete descriptions of) what is going on anywhere else at any time. In fact they tell me next to nothing about what is going on anywhere else at any time. The laws do not govern the evolution of the world in spacelike directions.

\footnotetext{
${ }^{10} \mathrm{I}$ am pretending here (for purposes of illustration) that Newtonian gravitational theory is deterministic, even though there are good arguments that it is not. Earman discusses these arguments in his [1986].

${ }^{11}$ As I've said, not every general relativistic spacetime can be partitioned into times. But the laws of general relativity still govern the evolution of worlds with those spacetimes in timelike directions at a local level: some four-dimensional regions of those spacetimes can be partitioned into times, and (if the region is the right shape) the laws determine what is happening at all times given information about what is happening at one time.
} 
I used the laws of Newtonian gravitational theory as an example, and these laws are deterministic. If some laws are deterministic, then given complete information about the state of the world on some time, those laws yield complete information about the state of the world on other times. Not all possible laws are deterministic; on some interpretations the laws of quantum mechanics are indeterministic. But these laws, together with complete information about the state of the world at a time, do assign probabilities to possible states at other times, and so do govern the world in timelike directions. They do not do the same for points of space.

Indeterministic laws, we usually think, must be probabilistic laws; and we think of probabilistic laws as assigning probabilities to possible future states of the world, but not to possible past states of the world. We think of these laws as governing the evolution of the world toward the future, but not in the opposite direction-toward the past. ${ }^{12}$ So while being a direction in which the laws govern the evolution of the world is sufficient for being a timelike direction, it is not necessary; the opposites of timelike directions are also timelike directions.

(There is another role that timelike directions play in some familiar laws that spacelike directions do not: quantities like mass, charge, and energy are conserved in timelike directions, but not in spacelike directions. But when there are conservation laws like this, they are usually derived from the dynamical laws (as, for example, the law of conservation of charge follows from Maxwell's equations-the dynamical laws for electromagnetism). So I need not explicitly mention this as a second role in the laws that distinguishes timelike from spacelike directions.)

It is not controversial that timelike directions play these roles in the laws with which we are most familiar. I propose that we take these roles as constitutive: what it is to be a timelike direction is to play these roles in the laws. To be explicit, the proposal is this:

\footnotetext{
${ }^{12}$ I talk about generic probabilistic laws here because the case of quantum mechanics is complicated. Even on interpretations of quantum mechanics that add a collapse postulate to Schrödinger's equation, the laws do govern the evolution of the world toward the past to some extent. Schrödinger's equation is time-reversal invariant, so the laws govern the evolution of the world toward the past back to the time of the most recent collapse of the wave function.
} 
Laws: A direction (that is either spatial or temporal according to the geometry) is timelike iff it (or its opposite) is a direction in which the laws govern the evolution of the world.

Let me make two remarks about this proposal.

First, I do not claim that it is necessary that the laws of nature govern the evolution of the world in some direction(s) in spacetime..$^{13}$ I do not claim, that is, that it is necessary that there be some timelike role in the laws to be filled. Perhaps there are possible worlds with strange laws of nature in which there is no such role. But I $d o$ claim that in such worlds no direction is a timelike direction.

Second, my proposal presupposes that the laws of nature are more fundamental than the distinction between timelike and spacelike directions. It presupposes, roughly, that it is possible to state the laws of nature without using the words 'time' and 'space.' For if in order to state the laws we had to presuppose that time and space had already been distinguished, then it would be going in a circle to then appeal to the laws to distinguish time from space. This is not a problem, though. We standardly state laws without appealing to the distinction between time and space. In formal presentations of, say, Newtonian gravitational theory, the distinction between timelike and spacelike directions is always made in some informal remarks after the author has described the geometrical structure of spacetime and before he writes down the equations of the theory. But the equations can be understood perfectly well with the informal remarks removed.

Or, to take a more concrete case, consider Newton's first law: a body not acted on by any forces moves with a constant velocity. 'Velocity' means the same as 'change in spatial location with respect to change in temporal location.' I claim that we can re-write this law to remove the reference to space and time. We can do this by using quantifiers: first, replace 'velocity' with 'change in location along the $x$ direction with respect to change in location along the $y$ direction'; then, preface the laws with the quantificational phrase, 'there are two (distinct) directions, $x$ and $y$, such that $x$ and $y$ play such-and-such geometrical roles, and....'

\footnotetext{
${ }^{13} \mathrm{I}$ 'm using 'the laws of nature' non-rigidly.
} 


\section{Testing Our Intuitions}

Let's take a look at a particular world with a two-dimensional spacetime and see if we agree that the laws distinguish space from time even though the geometry of spacetime does not. Consider the spacetime diagram in figure 4.

Figure 4:

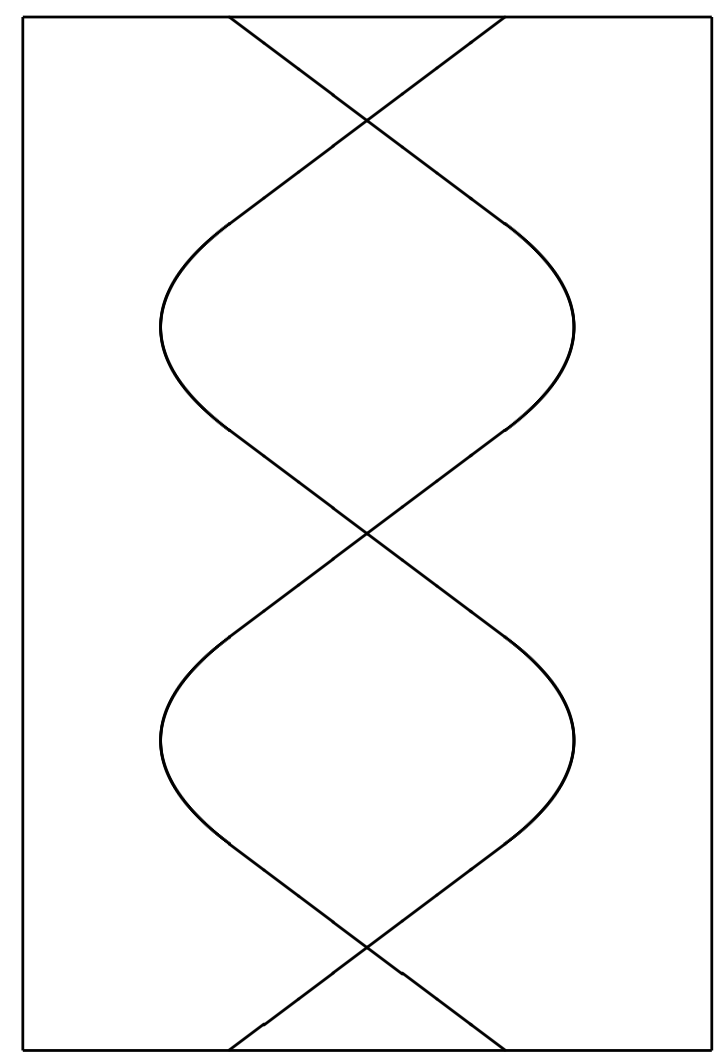

This diagram depicts the distribution of matter in spacetime in some possible world. (Suppose that spacetime has the familiar Newtonian structure, so that talk of space and time makes sense.) Normally we read the vertical axis of spacetime diagrams as the time axis. I ask you to forget about that convention for now and suppose you do not know which axis represents time. I contend that if you do not know what the laws are, you are unable to tell which axis is time. And that is evidence that it is the laws that are doing the work to make one axis time.

It certainly seems that there are possible worlds correctly represented by this 
diagram in which the vertical axis represents time; and also possible worlds correctly represented by this diagram in which the horizontal axis represents time. To make this plausible, let me describe for you one world of each kind. Call the world in which the vertical axis represents time 'the vertical world,' and the other, 'the horizontal world.' First, a description of the vertical world:

VERTICAL: there are two particles that accelerate toward each other, until they meet in an elastic collision and rebound back the way they came; then they slow down, turn around, and accelerate back toward each other, repeating this cycle for all time.

And here's a description of the horizontal world:

HoRIzONTAL: for a long time nothing happens. Then an infinite number of pairs of particles come into existence; one member of each pair moves off to the right, the other to the left. Each particle bounces off a particle coming from the other direction, then is annihilated in a collision with the particle with which it was created. Then nothing happens for the rest of time.

By itself the diagram doesn't favor one of these descriptions over the other.

Things are different, I think, when I tell you what laws of nature govern the world the diagram depicts. Let the laws be Newton's three laws of mechanics and a slightly amended version of Newton's law of universal gravitation. ${ }^{14}$ Of course Newton's laws contain terms like 'velocity' and 'acceleration' which are defined in terms of space and time; suppose them to be redefined in the way I mentioned above at the end of section 5 , so that velocity ends up being change in position along the

\footnotetext{
${ }^{14}$ According to Newton's law the force between two bodies is inversely proportional to the square of the distance between them. As the distance between two bodies goes to zero, the force gets infinitely large. A natural way to extend Newton's laws to deal with this case is to have the particles bounce off each other in a perfectly elastic collision.
} 
horizontal axis with respect to change in position along the vertical axis. ${ }^{15}$ So in these laws the vertical axis plays the time role in the definition of 'velocity', and also plays the role that I described above: the laws govern the evolution of the world along the vertical axis. It seems to me that when we add these laws to the description of the world depicted by the diagram, we know enough to know that the vertical axis is the time axis, and we rule out HorizonTAL as a correct description of that world.

Let me address two worries one might have about this example. First, one might worry that my strategy here-maintaining that knowing only how matter is distributed in spacetime, without knowing what the laws are, leaves us unable to tell which axis is time-is incoherent if a broadly empiricist theory of laws of nature is true. According to such theories, the laws of nature supervene on the occurrent (categorical, non-dispositional) facts. Since the occurrent facts surely include facts about the structure of spacetime and the distribution of matter in it, an empiricist will say that by fixing the facts about matter and spacetime I've already fixed what the laws are, and so already fixed which directions are timelike.

This may be so; I won't take a stand here on whether some empiricist theory of laws is true. But even if an empiricist theory is true, it is coherent to ask you to remain ignorant about the laws even though you know about the distribution of matter in spacetime, for it is not always obvious what the laws are in a world with a given set of occurrent facts. And the fact that we cannot tell which axis is

\footnotetext{
${ }^{15}$ So where the original laws contained '...velocity...' the new laws will say 'there are two (distinct) directions, $x$ and $y$, such that $x$ and $y$ play such-and-such geometrical roles, and....change in position in the $x$ direction with respect to change in position along the $y$ direction...'

Given that the horizontal and vertical axes play symmetric geometrical roles, you might wonder why it is the direction along the horizontal axis, rather than the direction along the vertical axis, that gets to play the time-role in the definition of velocity. But there is no answer to this question. The way the laws are stated guarantees that (in two-dimensional worlds with Newtonian spacetime) one kind of direction will play the time role in the definition of velocity and the other will not; but it is undetermined which - there are other worlds with the same spacetime geometry and the same laws in which the other plays that role.
} 
time when ignorant of the laws but can when we know the laws is still evidence that it is the role they play in the laws that makes timelike directions timelike. ${ }^{16}$ (In general, there is no incoherence in denying that lawhood is fundamental while using lawhood to analyze other non-fundamental notions.)

Second, one might worry about the descriptions in Vertical and Horizontal. Those descriptions seem to contain more information about the distribution of matter in spacetime than the spacetime diagram alone does. And the points at which the descriptions go beyond the information contained in the diagram are points at which the descriptions disagree. The spacetime diagram tells us only which spacetime points are occupied by material objects. The descriptions contain further information about how many material objects there are, and which points each object occupies. In Vertical I said that there were two particles, while in Horizontal I said there were infinitely many. Now, I claimed that knowing only how matter was distributed in spacetime isn't enough to allow us to figure out which axis is time. But one might complain that by presenting the spacetime diagram I hadn't given complete information about how matter is distributed in spacetime. Complete information requires the kind of information contained in Vertical and Horizontal. If I had said that there are only two particles, and each one occupies the points on just one of the curvy lines, then perhaps it would have seemed obvious that the vertical axis is time.

To allay this worry let me make some further stipulations about the world(s) represented by the diagram. I'll add information so Vertical and Horizontal won't contain additional information.

Suppose that in worlds represented by the diagram there are uncountably many material objects, that some of them are mereologically simple (without proper parts), and that each simple object occupies exactly one of the occupied spacetime points. Suppose also that for any collection of the simple objects there is a mere-

\footnotetext{
${ }^{16}$ An empiricist theory of laws entails that at most one of VerTical and Horizontal describes a possible world. For the descriptions disagree about which axis is time, and so (on my view) disagree about the laws, but agree on the occurrent facts. Empiricists should read the possibilities at work in my argument as epistemic, rather than metaphysical, possibilities.
} 
ologically complex object that they compose. (In slogans, then, I'm saying that (something like) four-dimensionalism and the doctrine of unrestricted mereological composition are true in these worlds.) Vertical and Horizontal seem to disagree about how many things there are and which regions those things occupy; but that is because the descriptions are not complete. They don't tell the stories of the spatiotemporal careers of every material object. Instead they only tell the stories of a few salient ones. When we move from Vertical to Horizontal we switch which axis we regard as the time axis; doing this brings about a shift in which of material objects are salient.

So far I've presented my proposal and given it some intuitive support. I will now discuss some objections to it.

\section{Symmetric Laws and Indeterminacy}

The claim that geometry distinguishes timelike from spacelike directions ran into problems with spacetimes in which timelike and spacelike directions play symmetric roles. I said that there is still a difference between timelike and spacelike directions in some of those worlds, and that it is their different roles in the laws which distinguishes them. But what about worlds in which timelike and spacelike directions play symmetric roles in both the geometry and the laws? There isn't any reason to deny that such laws are possible; there are even examples of such laws. The wave equation for a wave in one dimension, for example, is

$$
\frac{1}{v^{2}} \frac{\partial^{2} \phi}{\partial t^{2}}=\frac{\partial^{2} \phi}{\partial x^{2}}
$$

( $\phi$ is a function on spacetime; it tells you, intuitively speaking, 'how high' the wave is at each point of spacetime.) Now $v$ is the speed of the wave, and we're free to choose units in which it's 1 . In that case, the equation becomes

$$
\frac{\partial^{2} \phi}{\partial t^{2}}=\frac{\partial^{2} \phi}{\partial x^{2}}
$$


It is clear that in this law time and space play symmetric roles: switching $t$ and $x$ leaves the equation the same. Moreover, the roles time and space play in these laws both fit the description I gave above: these laws govern the evolution of the wave both along the time axis and along the space axis.

Laws entails that all directions in this world are timelike, and that is not right. There are three ways to respond to this problem. First, we could conclude that we do not yet have a complete account of what makes time different from space, and we could search for some other feature of the world that is doing work to distinguish spacelike from timelike directions. Second, we could conclude that it is just a brute fact that the timelike directions in this world are timelike, that there is nothing informative to be said about what makes them timelike. Or third, (paralleling the move from Dimension to Dimension*) we could conclude that if the geometry and laws fail to single out one kind of vector as the timelike vectors, then it is indeterminate which directions are timelike.

We should choose the third alternative. It does not seem that it could be just a brute fact which directions are timelike (I say more about the brute fact view below in section 9.3). And in these highly symmetric worlds it is hard to see what else, other than the geometry and the laws, could distinguish timelike from spacelike directions. Worlds governed by the wave equation look the same no matter which axis we regard as the time axis.

To deal with these symmetric worlds, then, amend Laws as follows:

Laws*: If a direction is timelike then the laws govern the evolution of the world along it (or its opposite); all vectors that point in timelike directions are of the same kind; and to the extent that these conditions fail to determine which vectors are timelike, it is to that extent indeterminate which vectors are timelike.

Laws and Laws* agree on all worlds except worlds like the one governed by the onedimensional wave equation. In such worlds Laws* entails that it is indeterminate which kind of vector is timelike.

How bad is it to admit that it is sometimes indeterminate which directions are timelike? Certainly it's perfectly determinate in our world which directions are 
timelike. Earlier I claimed that there are some two-dimensional worlds-complicated ones in which there is a lot going on-in which it is perfectly determinate which directions are timelike. But I don't think that this must be perfectly determinate in every two-dimensional world, so I see no problem accepting that it is indeterminate which directions are timelike in worlds in which time and space play symmetric roles in both the laws and the geometry of spacetime.

\section{Laws That Govern in a Spacelike Direction}

I turn now to a second objection. My view entails that it is not possible that there be laws that govern the evolution of the world is a spacelike direction. But (so the objection goes), surely this is possible. Surely we can produce examples of possible worlds with this feature.

I have yet to hear a convincing example. I will discuss four examples to give a sense of how I respond to examples of this kind. ${ }^{17}$

Example 1: In this world, it is a law that to the left of every apple is an orange, and to the left of every orange is an apple. (Suppose we've fixed, once and for all, which direction in space is to the left.) This law governs in a spacelike direction: if I know that there is an apple here, I get information about what is going on to the left.

Example 2: In this world, there is a special plane dividing space in half; and it is a law that the contents of space on one side of the plane are perfectly mirrored on the other side. This law governs in a spacelike direction: if I know that there is a red sphere in a certain place on one side of the plane, I get information about what is going on at the very same time in the corresponding place on the other side of the plane.

I am enough of a metaphysician to take examples like these somewhat seriously. I have three (independent) responses to them. First, I don't think the laws in these

\footnotetext{
${ }^{17}$ For helping me see the need to discuss examples of this kind, as well as for the examples themselves, I thank James van Cleve, Eric Lormand, Phillip Bricker, Joshua Schechter, and Jonathan Schaffer.
} 
examples are doing enough to govern the evolution of the world in a spacelike direction. Given complete information about what is going on at one place for all time, they do not give complete information about what is going on everywhere in some spatial direction at all times. (The law in example 1 gives only partial information (only information about the presence of apples or oranges) about what is going on to the left. The law in example 2 gives complete information, but only about just one other place for all time: the place that is the mirror-reflection of the initial place.) So they're not doing in a spacelike direction what laws like Newtonian mechanics do in a timelike direction.

Second, even if there is a world in which it is true that to the left of every apple is an orange (and so on), and a world with mirror symmetry, I'm not sure why I should believe that it would be a law that to the left of every apple is an orange, or that the world exhibits mirror symmetry. (Certainly the law about apples couldn't be a fundamental law.) For I find it hard to have intuitions about what the laws of some world are, given descriptions of the goings-on in those worlds.

Of course, some philosophers can argue that if there is a world in which to the left of every apple is an orange (and so on), and the world is simple enough in other ways, then it is a law that to the left of every apple is an orange. These are philosophers who accept an empiricist theory of laws. According to the most sophisticated empiricist theory - the best system theory of laws - those true statements are laws that are theorems of the deductive system that best balances simplicity and strength [Lewis 1986b]. If the apple world is simple enough in other ways, then the statement that to the left of every apple is an orange might make it in to the best system, and so might be a law.

I do not accept the best system theory of laws. So the conflict between my second response to examples 1 and 2 and the best system theory is not a problem for me. ${ }^{18}$ But I want to emphasize that philosophers who do accept the best system

\footnotetext{
${ }^{18}$ I do not just reject the theory because it conflicts with my theory of the difference between space and time. My primary reason for rejecting it is that it fails to respect our modal intuitions about lawhood. Briefly, empiricism about laws (and so the best system theory in particular) entails that there cannot be worlds that differ merely in what laws govern them. But I accept the counterexamples opponents of the best systems theory offer to this claim (for example, in [Carroll
} 
theory can still accept my account of what makes time different from space. As I mentioned above, my other arguments are neutral on whether some empiricist theory of laws is true. Empiricists just cannot use my second response to examples 1 and 2. My other responses are available to them.

I have a third response to examples like 1 and 2. It is part of the description of the possible worlds in these examples that certain directions are spacelike and certain others, timelike. But doesn't this beg the question? Why should we accept that the directions called 'spacelike' in the description really are spacelike?

One might refuse to answer this question. But then I am not sure why I should take seriously the claim that there are possible worlds like those described in the examples. Or, one might answer this question by proposing an alternative theory of what makes time different from space. I'll criticize a few such theories in the next section. Or, one might say: we can imagine the worlds described, and it is part of the content of our imagining that the directions called 'spacelike' are spacelike. And since imagination is a guide to possibility, that gives us reason to believe that these worlds are possible.

How does this act of imagination work? Maybe like this: we imagine watching the history of the world in question unfolding, as if we were watching a movie. We can tell which directions are timelike and which spacelike when watching a movie (without needing any theory of the difference to help us); in the same way, we can tell which directions are timelike and which spacelike in these worlds.

Imagining watching the history of the world unfold as if watching a movie is a legitimate way to learn which directions in that world are timelike only if those are the experiences an observer who existed in that world would have. But the laws we are given in example 1 and in example 2 are not rich enough to permit the existence of conscious observers. So we cannot use this procedure to defend the claim that the directions in which these laws (allegedly) govern the evolution of the world are spacelike directions.

The next two examples contain more realistic examples of laws.

Example 3: In this world, the laws are those of Newtonian mechanics and Newton's law of universal gravitation. This law governs in a space1994]). 
like direction: if I know that there is a particle here of a certain mass now, I know something about the value the gravitational field now has at every other point.

This example is not convincing. First, it is not clear that on a correct interpretation, Newtonian gravitational theory say that there really is any such thing as the gravitational field. And even if it does, that there is now a particle here of a certain mass, together with the laws, does not entail complete information about what is going on at any other place, much less at every other place. (Complete information about what is going on at some other place will tell us whether there are any particles there, not just what the value of the gravitational field is there.) That there is a particle here of a certain mass doesn't even entail what the value of the gravitational field is at every other point. To know that I'd have to know how many particles there were in total, and what their masses and positions are right now. If I know anything about what is going on elsewhere, it is only how the particle here contributes to the value of the gravitational field elsewhere; but this is consistent with the field's actual value being anything at all. So these laws do not govern in a spacelike direction.

Example 4: In this world, the laws of quantum mechanics govern the world. In an EPR-type experiment, there are two particles some distance apart, and if we measure the spin on one of them in some direction, we know with certainty the outcome of a measurement of the spin of the other particle in that same direction, even if the measurement events are spacelike-separated. So these laws govern the evolution of the world in a spacelike direction.

This example is not convincing. For laws to govern the evolution of the world in a spacelike direction, it must at least be the case that given complete information about what is going on right here, the laws give complete information about (or assign probabilities to complete description of) what is going on at other places that are spacelike separated from it. But if all we know is that (after being measured) some particle right here has spin-up in some direction, the laws don't tell us anything about what is going on elsewhere. They only give us information if we also know that there is another particle somewhere else, and that the 'system' 
comprising the two particles is in an entangled state. But this information is not just information about what is going on right here.

\section{Alternative Views}

Finally, I will review some alternative views about what makes time different from space, and say why I reject them. My goal is not to give these theories detailed formulations and refutations; I aim only to suggest why I think they go in the wrong direction from the start.

\subsection{The Causal Theory of Time}

According to this view, a direction is timelike just in case it is a possible direction of causation. This theory was inspired, I think, by a certain way of thinking about Minkowski spacetime. There is a synthetic axiomatization of this spacetime's geometry using just one two-place predicate that can be taken to mean ' $x$ and $y$ are causally connectible.' Perhaps this axiomatization is getting the metaphysics right: the spatiotemporal relations in Minkowski spacetime, and so facts about which events occur before which other events, are derived from a more fundamental relation of causal connectibility. ${ }^{19}$

I reject this theory for two reasons. The first, and less important reason, is that it precludes the possibility of instantaneous causation. It looks like Newtonian mechanics involves instantaneous causation-according to that theory the sun's being a certain distance from the earth instantaneously causes the earth to experience a certain force-and I accept that Newtonian worlds are metaphysically possible. But I place more importance on a second reason. I just don't think that facts about causation are more fundamental than the difference between space and time. But they have to be, for this theory to be right.

(My theory entails that the laws are more fundamental than the difference between space and time. One might wonder why I am comfortable with this and uncomfortable with the causal theory of time. I won't give a detailed answer to this question; I will just point out that many contemporary philosophers share the

\footnotetext{
${ }^{19}$ See [Sklar 1974].
} 
feeling that causation is less fundamental than lawhood. ${ }^{20}$ )

\subsection{Three-Dimensionalism}

Three-Dimensionalism is the view that material objects persist through time without having temporal parts. Since it is commonly admitted that material objects are extended in space by having spatial parts, there is an asymmetry here between space and time. One might try to distinguish space from time using this asymmetry: time is that dimension in which material objects are extended without being made up of parts.

Three-Dimensionalism is controversial, so insofar as I am not a three-dimensionalist I am not tempted by this proposal. But I do not think three-dimensionalists should be either. One standard argument against three-dimensionalism is the argument from temporary intrinsics: if something is spherical at a time, then it must have a temporal part that is spherical simpliciter, on pain of making sphericality a relation to times, and not an intrinsic property at all. ${ }^{21}$ Three-dimensionalists think they can resist this argument. But if they can, then they can also resist the parallel argument that material objects that are spatially extended must have spatial parts, the argument from local intrinsics: if something is red in one place and green in another, then it must have a spatial part that is red simpliciter, on pain of making redness a relation to places. (If you deny that redness and greenness are intrinsic properties choose other examples.) So three-dimensionalists ought to admit the possibility of material objects that are spatially extended without having any spatial parts. But once that possibility is granted, there is no longer an asymmetry between the way material objects are (or can be) extended in space and the way they are (or can be) extended in time.

\footnotetext{
${ }^{20}$ David Lewis [1986a] is one example: he analyzes causation in terms of counterfactuals, and his truth-conditions for counterfactuals appeal to laws. But even philosophers who reject counterfactual analyses of causation, like Maudlin [2004], believe that laws are more fundamental than causation.

${ }^{21}$ This argument is much discussed; it is presented, among other places, in [Lewis 1986b] and [Sider 2001].
} 


\subsection{The Brute Fact View}

According to the brute fact view, there is no need to appeal to geometry or the laws to distinguish spacelike from timelike directions. Instead, there is no way to distinguish spacetime from timelike directions in other terms. There is no informative answer to the question, 'what makes timelike directions timelike?'

One way to put this view is to say that there is a fundamental property of directions, the property of being a timelike direction. This property, being fundamental, has no analysis.

But it might seem odd to believe that things like directions could have fundamental properties. If we confine our attention to Newtonian spacetime we can avoid this oddness by avoiding talk of directions. Then the brute fact view looks like this: some regions of this spacetime are times, and others are not. The ones that are times have some fundamental intrinsic property that the others do not: the property of being a time. End of theory.

This theory is not plausible. Certainly the regions that have this special property must also play the appropriate role in the geometry. (Supposing we characterize Newtonian spacetime using two distance functions, the role is as follows: each region contains all and only the points that are zero distance from any point in that region, according to one of those distance functions.) But why is there this necessary connection between this special property and a certain geometrical role? The brute fact view gives no answer. My view does better: since it does not postulate the special property, it has no necessary connection to explain.

But maybe that is just a caricature of the brute fact view. Here is a closely related view one might have. Do not postulate a special non-geometrical fundamental property of being a time. Instead, pick out one of the geometrical relations that gives spacetime its structure, and make it special. Sticking to my focus on Newtonian spacetime, one way to put the view is as follows: this spacetime get its structure, let us suppose, from two distance functions. One of these is the temporal distance function, and the other is the spatial distance function. ${ }^{22}$ Times are regions

\footnotetext{
${ }^{22}$ There are analogous ways to formulate the view on other accounts of the fundamental spatiotemporal relations that characterize Newtonian spacetime.
} 
containing all and only the points that are zero distance from any point in them, according to the temporal distance function. What makes one function the temporal distance function, rather than the spatial distance function? There is no answer. It is just a brute fact.

This view I take more seriously as a competitor to my own than the others I have considered. But I do think it is wrong. The reason appeals, again, to twodimensional Newtonian spacetime.

Take a world $w$ with two-dimensional Newtonian spacetime, and 'rotate' all the matter in that world ' 90 degrees' 23 to produce a new world $w^{*}$. I can describe this world in a bit more detail, but I don't want to beg any questions by calling certain regions of spacetime in $w^{*}$ 'times' or 'points of space.' So I will have to pick out regions of spacetime in $w^{*}$ using features those regions have in $w$. The description 'regions of spacetime that are points of space in $w$ ' picks out a definite set of regions of spacetime in $w^{*}$, while leaving it open whether those regions are also points of space in $w^{*}$. In more detail, then, $w^{*}$ looks like this: events that are simultaneous in $w$ occur (in $w^{*}$ ) in regions that (in $w$ ) lie on different times but are in the same place. The laws of $w^{*}$ are also obtained from the laws of $w$ by 'rotation': the $w^{*}$-laws treat regions that are points of space in $w$ as the $w$-laws treat the regions that are instants of time in $w$.

I think $w^{*}$ is qualitatively indiscernible from $w$. But the brute fact view cannot say this. According to the brute fact view, two-dimensional Newtonian spacetime is not symmetric in this way. So the brute fact view entails that either the 'rotation' operations cannot be performed (that is, there is no such 'rotated' world), or, if they can, they result in a world that is very very different, qualitatively, from $w$. So I reject this view.

Both versions of the brute fact view are similar to the view that what makes the future different from the past is that the future direction in time has some special

\footnotetext{
${ }^{23}$ Of course, this doesn't really make sense in the geometry of Newtonian spacetime. What I really mean is: consider this world represented on a two-dimensional Euclidean plane, like a piece of paper; then rotate everything 90 degrees on this Euclidean plane; now consider the world this new diagram represents.
} 
intrinsic property that the past direction in time lacks. I reject the brute fact view for the same reason many reject this view about the difference between the past and the future. In the later case, it seems that a world in which the distribution of matter were 'mirror reversed' around a given time (the laws, being time-reverse invariant (we may suppose), would be the same) would not be a world in which everything were 'going backwards,' but a world in which the direction that is actually the future direction is the past direction. Many who hold this view identify the future direction with the direction in which entropy increases; so it is not intrinsic to the future direction that it $b e$ the future direction. I accept this view about the difference between the future and the past. ${ }^{24}$ And my view about the difference between space and time is analogous. ${ }^{25}$

\section{References}

Carroll, John W. (1994). Laws of Nature. New York: Cambridge University Press. Earman, John (1974). 'An Attempt to Add a Little Direction to 'The Problem of the Direction of Time'.' Philosophy of Science 41(1): 15-47.

— (1986). A Primer on Determinism. Boston: D. Reidel Publishing Company.

Lewis, David (1986a). 'Causation.' In Philosophical Papers, volume 2, 159-171. New York: Oxford University Press.

— (1986b). On The Plurality of Worlds. New York: Blackwell.

Maudlin, Tim (2002). 'Remarks on the Passing of Time.' Proceedings of the Aristotelian Society 102: 259-274.

\footnotetext{
${ }^{24}$ Maudlin [2002] and Earman [1974] defend the brute fact view about the difference between the future and the past. Price [1996] is one philosopher who rejects it. Reichenbach [1956] identifies the future with the direction of entropy increase.

${ }^{25}$ Thanks to Gordon Belot, Cian Dorr, Hartry Field, Pete Graham, and audiences at the 2004 APA Eastern division meeting and the Australian National University. I would also like to thank Tim Maudlin, my APA commentator.
} 
- (2004). 'Causation, Counterfactuals, and the Third Factor.' In John Collins, Ned Hall and L. A. Paul (eds.), Causation and Counterfactuals, chapter 18, 419-444. Cambridge, MA: MIT Press.

Price, Huw (1996). Time's Arrow and Archimedes' Point. New York: Oxford University Press.

Reichenbach, Hans (1956). The Direction of Time. Los Angeles: University of California Press.

Sider, Theodore (2001). Four-Dimensionalism. New York: Oxford University Press.

Sklar, Lawrence (1974). Space, Time, and Spacetime. Berkeley: University of California Press.

Williams, Donald C. (1951). 'The Myth of Passage.' The Journal of Philosophy 48(15): 457-472. 